\title{
Normal coordinate analysis of bilirubin vibrational spectra: effects of intramolecular hydrogen bonding
}

\author{
Bijun Yang, Robert C. Taylor and Michael D. Morris \\ Department of Chemistry, University of Michigan, Ann Arbor, MI 48109, U.S.A. \\ and \\ XIU-Zhen Wang, Jin-guang Wu, Bao-Zhu YU and Guang-XIAN Xu
Department of Chemistry, Peking University, Beijing, 100871, China
}

and

Roger D. Soloway

Division of Gastroenterology, University of Texas Medical Branch, Galveston TX 77550, U.S.A.

(Received 19 September 1992; accepted 24 January 1993)

\begin{abstract}
Normal coordinate analyses are presented for half-bilirubin molecules. Calculations for the A-B pyrromethenone include intramolecular hydrogen bonds, while those for the $C-D$ chromophore exclude intramolecular hydrogen bonds. Valence force-field parameters have been optimized to correlate closely with the IR and Raman spectra of the target molecules. The results of the calculations are compared with the spectra of bilirubin IXa and various model compounds in the solid state and solution.
\end{abstract}

\section{INTRODUCTION}

VIBRATIONAL spectroscopy is emerging as an important tool in the study of the structure of bilirubin in physiological and model matrices related to its role in liver pathology and in neonatal jaundice. Infrared microspectroscopy is increasingly used to examine the local structure of bilirubin in gallstones [1-3], both as a clinical diagnostic and as a probe of the mechanism of gallstone formation. Resonance Raman microspectroscopy has also been proposed as a diagnostic for bilirubin gallstones [4, 5]. Resonance Raman spectroscopy has been used to study changes in intramolecular hydrogen bonding in the bilirubin-albumin complex [6] and in bilirubin phospholipid complexes [7]. Even surface-enhanced Raman spectroscopy has been applied to the bilirubin-albumin complex system [8].

Bilirubin vibrational Raman spectroscopy has been impeded by lack of rigorous band assignments for the molecule. Early workers largely used group frequency correlations [9] from the standard IR spectroscopy literature. The Warshel-Daub procedure has been used to assign some of the bands of the bilirubin resonance Raman spectrum [10], but the agreement with experiment was not completely satisfactory and required the ad hoc assumption that the exo vinylpyrromethenone has a higher energy electronic transition than the endo vinylpyrromethenone in the acid form and that the order was reversed in the dianion. One of our laboratories has previously reported calculations for several bands in the aromatic ring mode and carbonyl stretching regions and have verified these with IR spectroscopy of the normal and deuterated bilirubins [11]. We have reported empirical assignments, largely correlations with the pyrrole or lactam moieties of the exo and endo vinylpyrromethenones, based on studies of an extensive series of model compounds $[6,7]$. Considerable disagreement exists amongst these studies as to the band assignments and calculated frequencies.

In the present study we present normal coordinate calculations of the vibrational modes of both structures of bilirubin, using a valence force field. We place special emphasis on the aromatic ring mode region and carbonyl stretching region ( $c a$ 
$\left.900-1700 \mathrm{~cm}^{-1}\right)$. These regions dominate the resonance Raman spectra and have been emphasized in most IR experiments as well. Calculations including and excluding the effects of intramolecular hydrogen bonds have been performed. We present new data on the spectra of deuterated bilirubins, as well as on new model compounds with perturbed propionic acid side chains.

It has long been appreciated that the solution chemistry of bilirubin is influenced by intramolecular hydrogen bonding. Similarly intramolecular hydrogen bonding should be an important aspect of the structures of the mixed bilirubin salts which are in bilirubin gallstones. Thus, both the solution and the solid phase hydrogen bonding are physiologically important.

\section{EXPERIMENTAL}

Mid-IR spectra of bilirubin (Sigma, reagent grade) were measured (Nicolet 7199B FTIR) at $2 \mathrm{~cm}^{-1}$ resolution in $\mathrm{KBr}$ pellets, fluorolube mulls and by photoacoustic spectroscopy of the pure solid. Far-IR $\left(20-200 \mathrm{~cm}^{-1}\right)$ spectra were measured at $4 \mathrm{~cm}^{-1}$ resolution using mineral oil or petroleum jelly mulls in polyethylene cells. Resonance Raman spectra of solid bilirubin were measured at $5 \mathrm{~cm}^{-1}$ resolution in $\mathrm{KBr}$ pellets using a double monochromator (Spex 1401), photon counting and argon in 488 or $514.5 \mathrm{~nm}$ excitation. FT-Raman spectra were taken with a Nicolet 910 spectrometer at $4 \mathrm{~cm}^{-1}$ resolution.

Deuterated bilirubin was prepared by replacement of the six labile protons by dissolution of solid bilirubin in $0.001 \mathrm{M} \mathrm{NaOH} / \mathrm{D}_{2} \mathrm{O}$ followed by reacidification in $1 \mathrm{M} \mathrm{DCl} / \mathrm{D}_{2} \mathrm{O}$. The deuterated solid was washed several times with $\mathrm{D}_{2} \mathrm{O}$ and $\mathrm{CDCl}_{3}$ and desiccated in a nitrogen atmosphere to remove residual solvent. Spectra of the perdeuterated compound were taken as fluorolube mulls.

The model compounds $\alpha, \alpha^{\prime}$-dimethylmesobilirubin (MBR) in the meso and racemic forms were prepared as described previously [12]. Resonance Raman spectra were obtained at a concentraion of $5 \times 10^{-4} \mathrm{M}$ in chloroform solvent. The resonance Raman spectra of these compounds were measured with Nd-YAG second harmonic $(532 \mathrm{~nm})$ excitation with a triple spectrograph/array detector instrument at $1 \mathrm{~cm}^{-1}$ resolution.

\section{Normal Coordinate Analyses}

\section{Structure}

Bilirubin IXa (structure 1) has two pyrromethenone rings (A-B or endo and C-D or exo) that lie in two planes $97^{\circ}$ apart. It contains 82 atoms in all. In the electronic ground state, there is no conjugation between the two rings. For simplicity, we use a model containing 44 atoms, which is just slightly larger than the A-B ring. However, we have calculated all of the modes of the A-B system, including the effect of the hydrogen bond, modeling them as shown in Fig. 1. The vibrational modes of this model will be the modes of a complete bilirubin molecule or the model compounds possessing the intramolecular hydrogen-bonded structure. Additionally, we have calculated the modes in the aromatic ring stretch and carbonyl ring stretching regions for the $\mathrm{C}-\mathrm{D}$ rings, omitting the hydrogen bonds. In the latter calculations, we also modeled the methyl and propionic side groups as their carbon atoms for simplicity. We used this simplified model to describe the bilirubins lacking intramolecular hydrogen bonds. This structure is observed in the bilirubin/sphingomyelin complex and in bilirubin/calcium salts [1-7]. The predictions can be tested against the published Raman spectra of vinylisoneoxanthobilirubinate, which has an exo-vinyl group.

The bond lengths and angles of bilirubin used to calculate Cartesian coordinates of pyrromethenone are from X-ray studies [13]. Diagrams of the molecules plotted in these Cartesian coordinates are shown in Figs 1 and 2. Force constants are taken from 2pyrrolidinone, pyrroles, lactams and related compounds [14-17]. For hydrogen bond stretching, a force constant of $0.05 \mathrm{mdyn} \AA^{-1}$, which is typical [18], was used. For hydrogen bond bending 0.005 mdyn $\AA^{-1}$ was used. 


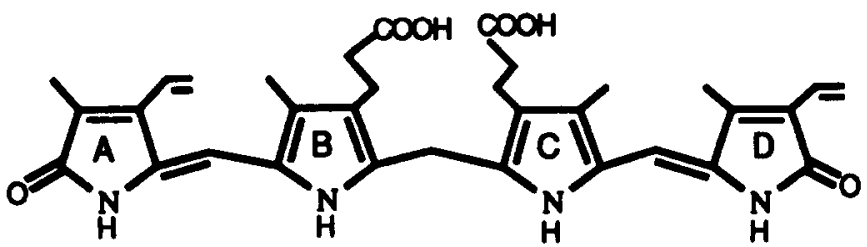

Structure 1

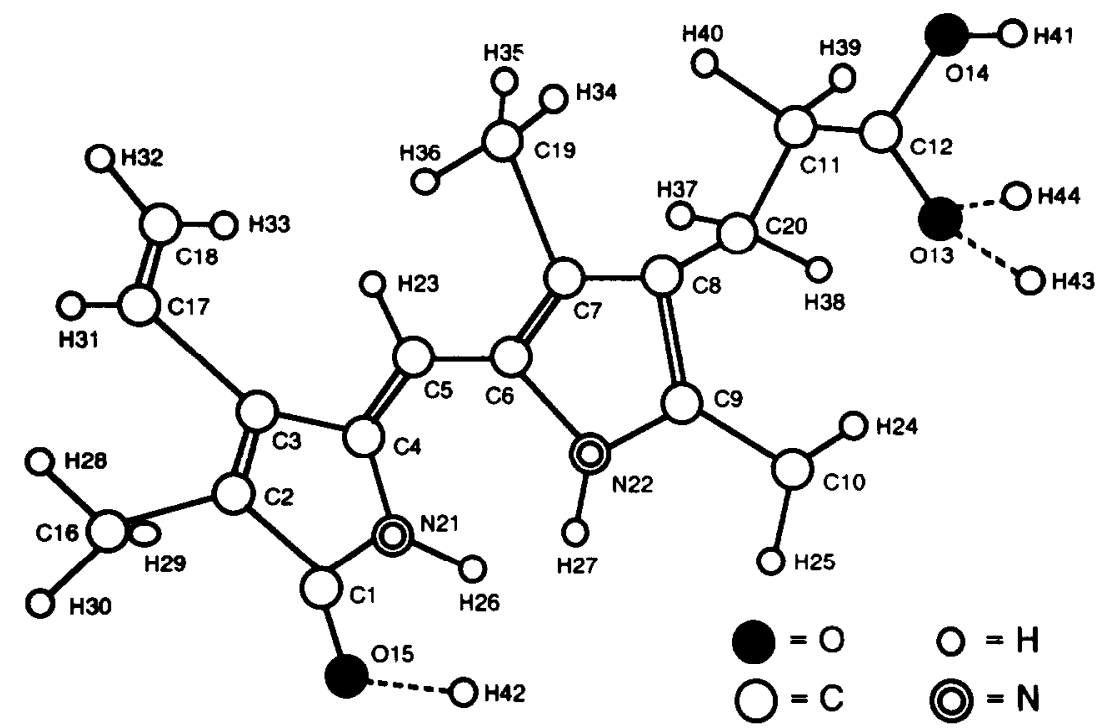

Fig. 1. Two-dimensional model of bilirubin IX $a$ (A-B) pyrromethenone.

\section{Method}

The general methods of normal coordinate analysis have been described in detail $[19$, 20]. The programs used in this work were written at the University of Michigan (C-D rings, interaction force constants, no hydrogen bonding) and Beijing University (A-B ring, hydrogen bonding) and are derived from classical public domain F-G matrix programs $[21,22]$. If we include the protons on the methyl and propionic acid side chains, there are 132 internal coordinates and 126 normal vibrations. Excluding these protons leaves 82 internal coordinates and 60 normal vibrations.

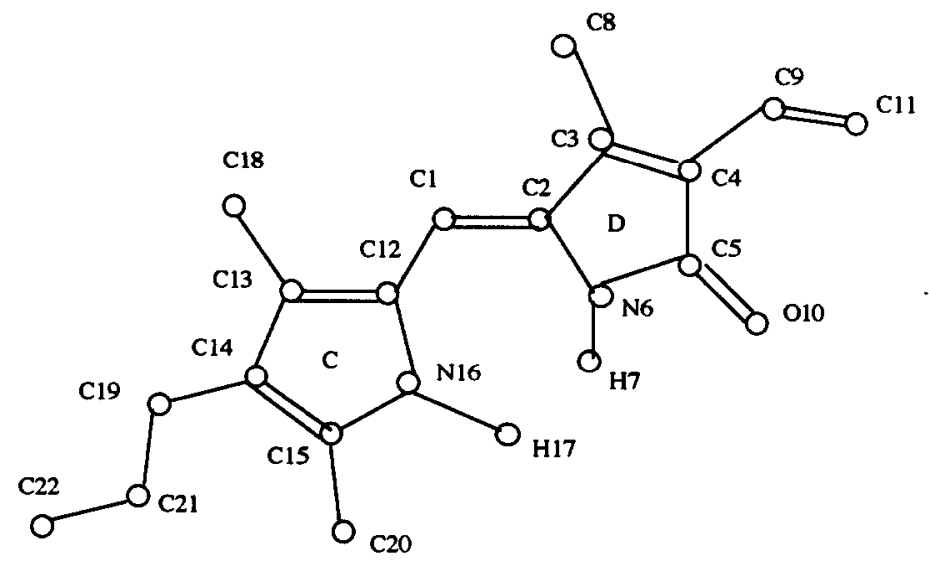

Fig. 2. Two-dimensional model of bilirubin IX $\alpha(C-D)$ pyrromethenone. 
Table 1. Internal coordinates and optimized force constants of bilirubin (AB) pyrromethenone

\begin{tabular}{|c|c|c|c|}
\hline No. & Internal coordinates & Vibration mode & Force constant \\
\hline \multicolumn{3}{|c|}{ Stretch } & $\operatorname{mdyn} \AA^{-1}$ \\
\hline 1. & $Q 1$ & $\mathrm{C}-\mathrm{O}(-\mathrm{H})$ & 8.12 \\
\hline 2. & $Q 2, Q 3$ & $\mathrm{C}-\mathrm{O}$ & 8.4 \\
\hline 3. & $Q 4$ & $\mathrm{~N}-\mathrm{C}$ (lactam) & 7.0 \\
\hline 4. & $Q 5, Q 6, Q 7$ & $\mathrm{~N}-\mathrm{C}$ & 3.0 \\
\hline 5. & $Q 8, Q 9, Q 10$ & $\mathrm{C}=\mathrm{C}$ (ring) & 7.6 \\
\hline 6. & $Q 11-Q 21$ & $\mathrm{C}-\mathrm{C}$ & 3.3 \\
\hline 7. & $Q 22, Q 23$ & $C=C$ (bridge) & 6.8 \\
\hline 8. & $Q 24-Q 29$ & $\mathrm{C}-\mathrm{H}\left(-\mathrm{CH}_{3}\right)$ & 4.67 \\
\hline 9. & $Q 30, Q 31$ & $\mathrm{C}-\mathrm{H}\left(\mathrm{C}=\mathrm{CH}_{2}\right)$ & 5.0 \\
\hline 10. & $Q 32-Q 37$ & $\mathrm{C}-\mathrm{H}\left(-\mathrm{CH}_{2}\right)$ & 4.76 \\
\hline 11. & $Q 38, Q 39$ & $\mathrm{C}-\mathrm{H}(-\mathrm{CH}=)$ & 5.1 \\
\hline 12. & $Q 40$ & N-H (lactam) & 5.82 \\
\hline 13. & $Q 41$ & $\mathrm{~N}-\mathrm{H}$ (pyrrole) & 6.37 \\
\hline 14. & $Q 42, Q 44, Q 45$ & $\mathrm{O}---\mathrm{H}$ ( $\mathrm{H}$ bonding) & 0.05 \\
\hline 15. & $Q 43$ & $\mathrm{O}-\mathrm{H}$ & 3.97 \\
\hline \multicolumn{3}{|c|}{ Bend } & $m d y n A ̉ r a d^{-2}$ \\
\hline 16. & $Q 46-Q 49, Q 62-Q 62, Q 66-Q 69$ & $(\angle \mathrm{CCC}(\mathrm{C}=\mathrm{C})$ & 0.7 \\
\hline 17. & $Q 50-Q 53, Q 65$ & $\angle \mathrm{CCN}$ & 0.5 \\
\hline 18. & $Q 54, Q 55$ & $\angle \mathrm{CNC}$ & 0.75 \\
\hline 19. & $Q 56, Q 65$ & $\angle \mathrm{OCN}$ & 0.5 \\
\hline 20. & $Q 57-Q 59$ & $\angle \mathrm{CCC}$ & 0.5 \\
\hline 21. & $Q 60, Q 61$ & $\angle \mathrm{HNC}$ & 0.41 \\
\hline 22. & $Q 70, Q 71$ & $\angle \mathrm{CCO}$ & 0.5 \\
\hline 23. & $Q 72$ & $\angle \mathrm{OCO}$ & 0.5 \\
\hline 24. & $Q 73-Q 78$ & $\angle \mathrm{CCH}\left(-\mathrm{CH}_{3}\right)$ & 0.37 \\
\hline 25. & $Q 79-Q 82, Q 85, Q 88$ & $\angle \mathrm{HCH}$ & 0.50 \\
\hline 26. & $Q 83, Q 84$ & $\angle \mathrm{CCH}$ (vinyl $-\mathrm{CH}_{2}$ ) & 0.45 \\
\hline 27. & $Q 86, Q 87$ & $\angle \mathrm{CCH}$ (bridge) & 0.50 \\
\hline 28. & $Q 89-Q 96$ & $\angle \mathrm{CCH}$ (vinyle) & 0.49 \\
\hline 29. & $Q 97-Q 100$ & $\angle \mathrm{CCH}$ (bridge) & 0.59 \\
\hline 30. & $Q 101-Q 104$ & $\angle \mathrm{COH}$ & 0.552 \\
\hline 31. & $Q 105$ & $\angle \mathrm{CO}---\mathrm{H}$ & 0.005 \\
\hline \multicolumn{4}{|c|}{ Torsion } \\
\hline 32. & $Q 106-Q 124$ & $\begin{array}{l}\text { Groups connected with lactam or } \\
\text { pyrrole, or on the rings, } \\
\text { e.g. } \mathrm{C}_{5} \mathrm{C}_{4} \mathrm{~N}_{21} \mathrm{C}_{1}\end{array}$ & 0.24 \\
\hline \multicolumn{4}{|c|}{ Wagging } \\
\hline 33. & $Q 125-Q 132$ & $\begin{array}{l}\text { Out-of-plane vibrations, } \\
\text { e.g. } \mathrm{O}_{15}-\mathrm{C}_{1} \mathrm{C}_{2} \mathrm{~N}_{21}\end{array}$ & 0.11 \\
\hline
\end{tabular}

Table 1 lists the internal coordinates and optimized force constants used in the detailed A-B calculations. Table 2 lists the internal coordinates and force constants used in the C-D ring calculations.

\section{Results AND Discussion}

Table 3 lists the calculated and observed frequencies for the A-B pyrromethenone obtained from the detailed calculations, as well as experimental IR and Raman frequencies obtained for solid bilirubin. Table 4 lists aromatic ring and carbonyl frequencies for the C-D pyrromethenone obtained from the simplified model and the corresponding experimental values from vinylisoneoxanthobilirubinate. Selected IR frequencies of the perdeuterated compound are listed in Table 5.

The bands in the low frequency region are wagging, torsion and bending modes of bilirubin. We present them for the A-B ring system only. The agreement between calculated and measured frequencies is good in this region. 
Table 2. Internal coordinates and valence force constants of bilirubin (C-D) pyrromethenone

\begin{tabular}{|c|c|c|c|}
\hline No. & Internal coordinates & $\begin{array}{l}\text { Major force constants } \\
\text { Vibration mode }\end{array}$ & Force constant \\
\hline \multicolumn{3}{|c|}{ Stretch } & $\operatorname{mdyn} A^{-1}$ \\
\hline 1. & $Q 1$ & $\mathrm{C}=\mathrm{C}$ (bridge) & 8.212 \\
\hline 2. & $Q 2, Q 4$ & $C-C$ (lactam) & 4.174 \\
\hline 3. & $Q 3$ & $\mathrm{C}=\mathrm{C}($ lactam $)$ & 8.653 \\
\hline 4. & 05 & $\mathrm{~N}-\mathrm{C}(=\mathrm{O})$ & 5.623 \\
\hline 5. & $Q 6, Q 16, Q 80$ & $\mathrm{C}-\mathrm{N}(=\mathrm{C})$ & 7.695 \\
\hline 6. & $Q 7, Q 8, Q 18, Q 19$ & $C-C^{*}(\beta)$ & 4.743 \\
\hline 7. & $Q 9$ & $\mathrm{C}=\mathrm{O}$ & 11.795 \\
\hline 8. & $Q 10$ & N-H (lactam) & 6.711 \\
\hline 9. & $Q 11, Q 13, Q 15$ & $\mathrm{C}=\mathrm{C}$ (vinyl, pyrrole $)$ & 8.366 \\
\hline 10. & $Q 12, Q 20$ & $C-C^{*}(\alpha)$ & 4.756 \\
\hline 11. & $Q 14$ & C-C (pyrrole) & 5.999 \\
\hline 12. & $Q 17$ & $\mathrm{~N}-\mathrm{H}$ (pyrrole) & 6.864 \\
\hline 13. & $Q 21, Q 22$ & $\mathrm{C}-\mathrm{C}$ (propionic) & 4.802 \\
\hline \multicolumn{3}{|c|}{ Bend } & $\operatorname{mdyn} A \mathrm{rad}^{-2}$ \\
\hline 14. & $Q 23, Q 40, Q 51$ & $\angle \mathrm{CCC}^{*}(a)$ & 0.620 \\
\hline 15. & $Q 24, Q 41, Q 52$ & $\angle \mathrm{NCC}^{*}$ & 0.620 \\
\hline 16. & $Q 25, Q 28, Q 42, Q 45$ & $\angle \mathrm{CCN}$ (ring) & 1.277 \\
\hline 17. & $Q 26, Q 27, Q 43, Q 44$ & $\angle \mathrm{CCC}$ & 1.277 \\
\hline 18. & $Q 29, Q 46$ & $\angle \mathrm{CNC}$ & 1.671 \\
\hline 19. & $Q 30-Q 33, Q 47-Q 50$ & $\angle \mathrm{CCC}^{*}(\beta)$ & 0.595 \\
\hline 20. & $Q 34$ & $\angle \mathrm{OCC}$ & 0.945 \\
\hline 21. & $Q 35$ & $\angle O C N$ & 0.945 \\
\hline 22. & $Q 36, Q 37$ & $\angle H N C$ (lactam) & 0.411 \\
\hline 23. & $Q 38, Q 39$ & $\angle C C C$ (vinyle, bridge) & 0.795 \\
\hline 24. & $Q 53, Q 54$ & $\angle \mathrm{HNC}$ (pyrrole) & 0.438 \\
\hline 25. & $Q 55, Q 56$ & $\angle C C C$ (propionic) & 1.032 \\
\hline \multicolumn{4}{|c|}{ Wagging } \\
\hline 26. & $Q 57, Q 61, Q 64$ & $\mathrm{C}^{*}(\alpha)-\mathrm{CCN}$ & 0.409 \\
\hline 27. & $Q 58, Q 59, Q 62, Q 63$ & $C^{*}(\beta)-\mathrm{CCC}$ & 0.509 \\
\hline 28. & $Q 60$ & $\mathrm{O}-\mathrm{CCN}$ & 0.686 \\
\hline 29. & Q65 & H-NCC (pyrrole) & 0.121 \\
\hline 30. & $Q 66$ & N-NCC (lactam) & 0.140 \\
\hline 31. & $Q 67$ & $\mathrm{C}-\mathrm{CCC}$ (vinyle) & 0.509 \\
\hline 32. & $Q 68, Q 69$ & $\mathrm{C}-\mathrm{CCC}$ (propionic) & 0.500 \\
\hline \multicolumn{4}{|c|}{ Torsion } \\
\hline 33. & $Q 70-Q 79$ & $\begin{array}{l}\text { Groups connected with lactam or } \\
\text { pyrrole, or on the rings, e.g. } \\
\mathrm{N}_{6} \mathrm{C}_{2}-\mathrm{C}_{3} \mathrm{C}_{4}\end{array}$ & 1.270 \\
\hline 34. & $Q 81$ & $\mathrm{~N}_{6} \mathrm{C}_{2}-\mathrm{C}_{1} \mathrm{C}_{12}$ & 0.579 \\
\hline 35. & $Q 82$ & $\mathrm{~N}_{16} \mathrm{C}_{12}-\mathrm{C}_{1} \mathrm{C}_{2}$ & 0.041 \\
\hline \multicolumn{4}{|c|}{ Interaction force constants } \\
\hline \multicolumn{4}{|c|}{ Stretch-stretch (pyrrole ring) } \\
\hline \multirow[t]{2}{*}{36.} & & $\mathrm{C}=\mathrm{C}, \mathrm{C}-\mathrm{C}$ & 0.764 \\
\hline & & $\mathrm{C}=\mathrm{C}, \mathrm{C}-\mathrm{N} ; \mathrm{C}$ common & \\
\hline 37. & & $\mathrm{C}=\mathrm{C}, \mathrm{C}=\mathrm{C}$ & -0.764 \\
\hline 38. & & $\mathrm{C}-\mathrm{C}, \mathrm{C}-\mathrm{N}$ & -0.791 \\
\hline 39. & & $\mathrm{C}=\mathrm{C}, \mathrm{C}-\mathrm{N} ; \mathrm{C}$ not common & -0.349 \\
\hline \multicolumn{4}{|c|}{ Stretch-stretch (lactam ring) } \\
\hline 40. & & $\mathrm{C}-\mathrm{C}, \mathrm{C}=\mathrm{C}$ & 0.256 \\
\hline 41. & & $\mathrm{C}-\mathrm{C}, \mathrm{C}-\mathrm{C}$ & 0.155 \\
\hline 42. & & $\mathrm{C}-\mathrm{C}, \mathrm{C}-\mathrm{N} ; \mathrm{C}$ not common & -0.065 \\
\hline
\end{tabular}


Table 2. continued

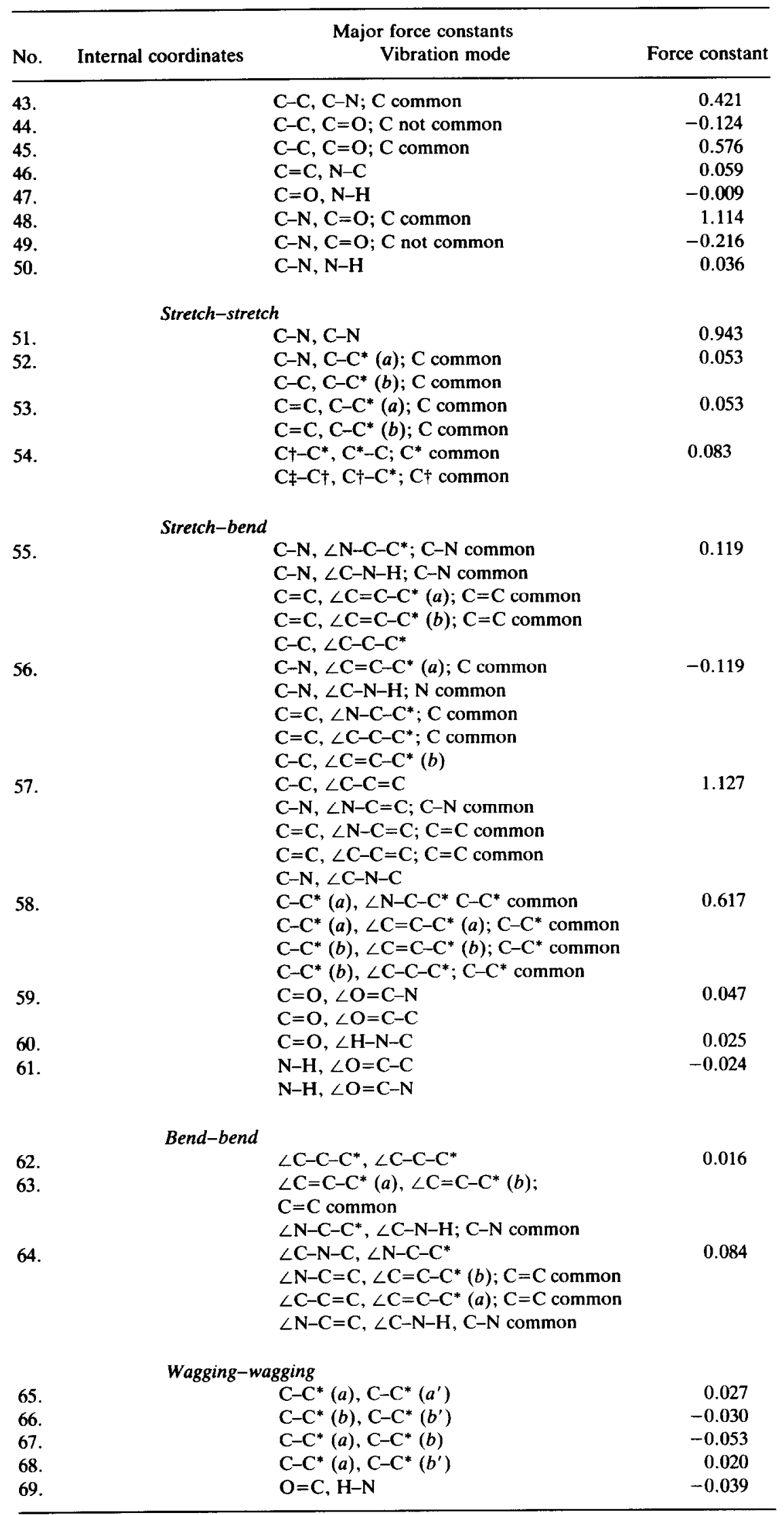


Table 2. continued

\begin{tabular}{|c|c|c|c|}
\hline \multirow[b]{2}{*}{ No. } & \multicolumn{3}{|c|}{ Major force constants } \\
\hline & Internal coordinates & Vibration mode & Force constant \\
\hline \multicolumn{4}{|c|}{ Wagging-torsion } \\
\hline 70. & & $\mathrm{C}-\mathrm{C}^{*}(a), t(a b)$ & 0.314 \\
\hline 71. & & $\mathrm{C}-\mathrm{C}^{*}(b), t(a b)$ & -0.296 \\
\hline 72. & & $\mathrm{C}-\mathrm{C}^{*}(a), t\left(a^{\prime} b^{\prime}\right)$ & -0.068 \\
\hline 73. & & $\mathrm{C}-\mathrm{C}^{*}(b), t\left(a^{\prime} b^{\prime}\right)$ & -0.090 \\
\hline \multicolumn{4}{|c|}{ Torsion-torsion } \\
\hline 74. & & $t(a b), t\left(a^{\prime} b^{\prime}\right)$ & -0.229 \\
\hline
\end{tabular}

* Methyl carbon attached directly to rings.

† One carbon away from $\mathrm{C}^{*}$.

$\$$ Two carbons away from $C^{*}$.

In the $900-1700 \mathrm{~cm}^{-1}$ region many modes are strongly resonance enhanced. This region has been emphasized in resonance Raman studies and has several strong and empirically useful IR bands as well. We discuss each of the bands in detail.

The hydrogen-bonded A-B calculation places a $965 \mathrm{~cm}^{-1}$ vibration on the pyrrole ring. It is the stretching of the methyl carbon connected to the pyrrole, and a stretching of $\mathrm{C}(9)-\mathrm{N}(22)$. The non-hydrogen bonded $\mathrm{C}-\mathrm{D}$ model predicts a mode at $945 \mathrm{~cm}^{-1}$, which describes a stretch extending to the lactam moiety. In solution, this mode has previously been observed to be strongly hydrogen bond dependent $[6,7]$. Thus, the extended assignment conforms more closely to the environmental sensitivity.

The A-B model calculation localizes $995 \mathrm{~cm}^{-1}$ as a lactam vinyl group bending. In the resonance Raman spectrum there is a strong band at $997 \mathrm{~cm}^{-1}$ in bilirubin XIII $a$ (A-B rings only). This band is relatively stable to the hydrogen bonding changes and shifts about $10 \mathrm{~cm}^{-1}$ in MBR XIII $\alpha$, in which the vinyl groups are replaced by ethyl groups. The assignment is realistic. There is, in addition, a propionic acid side $\mathrm{C}-\mathrm{O}-\mathrm{H}$ bending mode predicted at $982 \mathrm{~cm}^{-1}$. This would be observed in the IR but would not be seen in the resonance Raman spectra.

The non-hydrogen-bonded model predicts a torsion of the exo $\mathrm{C}=\mathrm{C}$ group connecting the lactam and pyrrole moieties and bending of the vinyl group at $1032 \mathrm{~cm}^{-1}$ for the C-D ring. The band at about $1032 \mathrm{~cm}^{-1}$ is weak in vinylisoneoxanthobilirubinate.

In both sets of calculations the band in the $1060 \mathrm{~cm}^{-1}$ region is assigned largely to a pyrrole ring stretching mode. In the resonance Raman spectra of all model compounds reported, including the monomeric forms, there is a medium or strong hydrogen bond insensitive band in the $1050-1060 \mathrm{~cm}^{-1}$ region, confirming the pyrrole assignment.

The more detailed A-B model finds propionic acid methylene bending $\mathrm{C}-\mathrm{H}$ bending modes at 1087 and $1106 \mathrm{~cm}^{-1}$, which agree well with the 1092 and $1107 \mathrm{~cm}^{-1}$ bands observed in the bilirubin IR and Raman spectrum.

The non-hydrogen-bonded model also predicts a $C-D$ vinyl $C=C$, exo $C=C$ and lactam, pyrrole $\mathrm{C}-\mathrm{N}$ stretch at $1107 \mathrm{~cm}^{-1}$. This band is not well resolved from the strong and broad $1056 \mathrm{~cm}^{-1}$ band in the monomeric compounds but is a medium-to-strong peak in the spectra of bilirubin III $\alpha$, XIII $\alpha$ and IX $\alpha$. The mode composition may be fairly realistic, because it shifts about $5 \mathrm{~cm}^{-1}$ or more from bilirubin III $\alpha$ (bilirubin XIII $\alpha$ ) to meso bilirubin XIII $\alpha$ where the vinyl group is replaced by an ethyl group. This strong vinyl group sensitivity confirms our assignment.

The simplified model calculates a pyrrole in-plane methyl deformation at $1144 \mathrm{~cm}^{-1}$ in the $\mathrm{C}-\mathrm{D}$ system. We equate this band with the band observed in the Raman spectrum at $1141 \mathrm{~cm}^{-1}$ in vinylisoneoxanthobilirubinate aqueous solution. This assignment may be a good one since this band appears to have a big shift or be a new band peculiar to the half bilirubin molecule. The significant changes in the spectra, in this case, should affect the pyrrole ring more than that of the lactam ring.

The A-B model predicts a vinyl ethylenic stretching and bending mode of the lactam at $1173 \mathrm{~cm}^{-1}$, which is equated to the $1164 \mathrm{~cm}^{-1}$ band in the IR $\left(1180 \mathrm{~cm}^{-1}\right.$, weak, in the 
Table 3. Calculated and observed frequencies and potential energy distribution PED

\begin{tabular}{|c|c|c|c|c|}
\hline \multirow[b]{2}{*}{ Calcd } & \multicolumn{2}{|c|}{ Raman } & \multirow[b]{2}{*}{ IR } & \multirow[b]{2}{*}{ Assignment and PED (\%) } \\
\hline & $\mathbf{R R}$ & FT-R & & \\
\hline 3409 & & & 3410 & $\mathbf{N}_{22}-\mathbf{H}_{27} 1.0$ \\
\hline 3260 & & & 3262 & $\mathbf{N}_{21}-\mathrm{H}_{26} 1.0$ \\
\hline 3101 & & 3097 & 3101 & $\mathrm{C}_{18}-\mathrm{H}_{33} .49, \mathrm{Cl}_{8} \mathrm{H}_{32} .48$ \\
\hline 3071 & & 3070 & 3070 & $\mathrm{C}_{5}-\mathrm{H}_{23} .99$ \\
\hline 3002 & & 3007 & 3011 & $\mathrm{C}_{20}-\mathrm{H}_{38} .31, \mathrm{C}_{20}-\mathrm{H}_{37} .29, \mathrm{C}_{11}-\mathrm{H}_{40} .21, \mathrm{C}_{11}-\mathrm{H}_{39} .19$ \\
\hline 2968 & & 2965 & 2968 & $\mathrm{C}_{19}-\mathrm{H}_{36} .69, \mathrm{C}_{19}-\mathrm{H}_{35} .17, \mathrm{C}_{19}-\mathrm{H}_{34} .13$ \\
\hline 2924 & & 2918 & 2913 & $\mathrm{C}_{10}-\mathrm{H}_{24} .49, \mathrm{C}_{10}-\mathrm{H}_{25} .50$ \\
\hline 2857 & & 2852 & 2858 & $\mathrm{C}_{19}-\mathrm{H}_{34} .35, \mathrm{C}_{19}-\mathrm{H}_{35} .35, \mathrm{C}_{19}-\mathrm{H}_{36} .31$ \\
\hline 2680 & & & 2673 & $\mathrm{O}_{14}-\mathrm{H}_{41} 1.0$ \\
\hline 1688 & 1680 & & 1692 & $\mathrm{C}_{12}-\mathrm{O}_{14}, .42 \mathrm{C}_{12}-\mathrm{O}_{13} .47$ \\
\hline 1655 & 1637 & 1650 & 1649 & $\mathrm{C}_{1}-\mathrm{O}_{15} .36, \mathrm{~N}_{21}-\mathrm{C}_{1} .17, \mathrm{C}_{3}-\mathrm{C}_{2} .13$ \\
\hline 1606 & 1614 & 1610 & 1612 & $\mathrm{C}_{3} \mathrm{C}_{2} .26, \mathrm{C}_{1}-\mathrm{O}_{15} .15, \mathrm{C}_{6}-\mathrm{C}_{7} .13, \mathrm{~N}_{21}-\mathrm{C}_{1} .10$ \\
\hline 1567 & 1569 & 1570 & 1571 & $\mathrm{C}_{6}-\mathrm{C}_{7} .30, \mathrm{C}_{4}-\mathrm{C}_{5} .21, \mathrm{C}_{3}-\mathrm{C}_{2} .09$ \\
\hline 1487 & 1504 & 1502 & 1500 & $\mathrm{H}_{32} \mathrm{C}_{18} \mathrm{H}_{33} .22, \mathrm{H}_{31} \mathrm{C}_{17} \mathrm{C}_{18} .21, \mathrm{H}_{31} \mathrm{C}_{17} \mathrm{C}_{3} .17$ \\
\hline 1448 & 1456 & 1451 & 1441 & $\mathrm{C}_{11}-\mathrm{C}_{12} .27, \mathrm{C}_{12}-\mathrm{O}_{13} .22, \mathrm{C}_{12}-\mathrm{O}_{14} .23$ \\
\hline 1395 & 1395 & 1404 & 1405 & $\mathrm{~N}_{21}-\mathrm{C}_{1} .17, \mathrm{H}_{28} \mathrm{C}_{16} \mathrm{H}_{30} .11, \mathrm{C}_{1}-\mathrm{C}_{2} .13$ \\
\hline 1356 & 1365 & 1363 & 1364 & $\mathrm{H}_{28} \mathrm{C}_{16} \mathrm{H}_{30} .12, \mathrm{H}_{35} \mathrm{C}_{19} \mathrm{H}_{36} .11, \mathrm{H}_{34} \mathrm{C}_{19} \mathrm{H}_{36} .11$ \\
\hline 1353 & 1340 & 1340 & 1344 & $\mathrm{H}_{35} \mathrm{C}_{19} \mathrm{H}_{36} .11, \mathrm{H}_{34} \mathrm{C}_{19} \mathrm{H}_{36} .10$ \\
\hline 1310 & 1291 & 1290 & 1301 & $\mathrm{H}_{23} \mathrm{C}_{5} \mathrm{C}_{4} .24, \mathrm{C}_{4}-\mathrm{C}_{5} .20, \mathrm{~N}_{21}-\mathrm{C}_{1} .15, \mathrm{H}_{23} \mathrm{C}_{5} \mathrm{C}_{6} .16$ \\
\hline 1243 & 1248 & 1245 & 1250 & $\mathrm{C}_{20} \mathrm{C}_{11} \mathrm{H}_{39} .14, \mathrm{H}_{39} \mathrm{C}_{11} \mathrm{C}_{12} .10, \mathrm{C}_{7}-\mathrm{C}_{8} .10$ \\
\hline 1225 & & & 1220 & $\mathrm{C}_{3}-\mathrm{C}_{4} .19, \mathrm{C}_{5}-\mathrm{C}_{17} .16, \mathrm{H}_{31} \mathrm{C}_{17} \mathrm{C}_{3} .09$ \\
\hline 1187 & 1190 & 1191 & 1186 & $\mathrm{~N}_{22}-\mathrm{C}_{9} .13, \mathrm{C}_{\tau}-\mathrm{C}_{8} \cdot 11, \mathrm{~N}_{22}-\mathrm{C}_{6} .08$ \\
\hline 1173 & 1168 & 1160 & 1164 & $\mathrm{C}_{17}-\mathrm{C}_{18} .41, \mathrm{H}_{31} \mathrm{C}_{17} \mathrm{C}_{18} .12, \mathrm{C}_{17} \mathrm{C}_{18} \mathrm{H}_{33} .09$ \\
\hline 1106 & & & 1107 & $\begin{array}{l}\mathrm{C}_{11} \mathrm{C}_{12} \mathrm{H}_{37} .17, \mathrm{C}_{8} \mathrm{C}_{20} \mathrm{H}_{37} .16, \mathrm{C}_{11} \mathrm{C}_{20} \mathrm{H}_{38} .14 \\
\mathrm{C}_{8} \mathrm{C}_{20} \mathrm{H}_{38} .12, \mathrm{C}_{20} \mathrm{C}_{11} \mathrm{H}_{39} .10\end{array}$ \\
\hline 1087 & 1096 & 1088 & & $\begin{array}{l}\mathrm{H}_{40} \mathrm{C}_{11} \mathrm{C}_{12} .19, \mathrm{C}_{20} \mathrm{C}_{11} \mathrm{H}_{40} \cdot 18, \mathrm{H}_{39} \mathrm{C}_{11} \mathrm{C}_{12} \cdot 14 \\
\mathrm{C}_{20} \mathrm{C}_{11} \mathrm{H}_{39} .12\end{array}$ \\
\hline 1057 & 1048 & 1060 & 1050 & $\mathrm{C}_{9}-\mathrm{C}_{10} .17, \mathrm{~N}_{22}-\mathrm{C}_{9} .15, \mathrm{C}_{5}-\mathrm{C}_{6} .10, \mathrm{C}_{T}-\mathrm{C}_{8} .10$ \\
\hline 1015 & & 1009 & & $\begin{array}{l}\mathrm{N}_{21}-\mathrm{C}_{4} .15, \mathrm{C}_{2}-\mathrm{C}_{16} .15, \mathrm{~N}_{22}-\mathrm{C}_{6} .08, \mathrm{H}_{26} \mathrm{~N}_{21} \mathrm{C}_{1} .11, \\
\mathrm{C}_{1}-\mathrm{O}_{15} .10\end{array}$ \\
\hline 995 & 988 & 992 & 994 & $\mathrm{C}_{17} \mathrm{C}_{18} \mathrm{H}_{32} .29, \mathrm{C}_{17} \mathrm{C}_{18} \mathrm{H}_{33} .27$ \\
\hline 982 & & & 983 & $\mathrm{C}_{12} \mathrm{O}_{14} \mathrm{H}_{41} .88$ \\
\hline 965 & 948 & 950 & 935 & $\mathrm{C}_{8}-\mathrm{C}_{20} .17, \mathrm{C}_{9} \mathrm{C}_{10} \mathrm{H}_{24} .16, \mathrm{C}_{\tau}-\mathrm{C}_{19} .12, \mathrm{~N}_{22}-\mathrm{C}_{9} .09$ \\
\hline 905 & & 900 & & $\mathrm{C}_{9} \mathrm{C}_{10} \mathrm{H}_{24} .23, \mathrm{C}_{9} \mathrm{C}_{10} \mathrm{H}_{25} .18$ \\
\hline 835 & 845 & 835 & 844 & $\mathrm{C}_{2} \mathrm{C}_{16} \mathrm{H}_{30} .29, \mathrm{C}_{2} \mathrm{C}_{16} \mathrm{H}_{28} .23$ \\
\hline 826 & 825 & & 820 & $\mathrm{C}_{7} \mathrm{C}_{19} \mathrm{H}_{34} \cdot 15$ \\
\hline 796 & & 790 & 790 & $\mathrm{H}_{27} \mathrm{~N}_{22} \mathrm{C}_{6} .11 \mathrm{C}_{2} \mathrm{C}_{16} \mathrm{H}_{29} .24$ \\
\hline 729 & 725 & 738 & & $\mathrm{C}_{7} \mathrm{C}_{19} \mathrm{H}_{34} .08$ \\
\hline 700 & 700 & & 698 & $\begin{array}{l}\mathrm{H}_{26}-\mathrm{N}_{21} \mathrm{C}_{1} \mathrm{C}_{4} .42, \mathrm{C}_{3} \mathrm{C}_{5} \mathrm{C}_{4}-\mathrm{N}_{21} \mathrm{H}_{26} \mathrm{C}_{1} .25 \\
\mathrm{O}_{15} \mathrm{C}_{2} \mathrm{C}_{1}-\mathrm{N}_{21} \mathrm{C}_{4} \mathrm{H}_{26} .23\end{array}$ \\
\hline 665 & & & 669 & $\mathrm{H}_{23}-\mathrm{C}_{5} \mathrm{C}_{4} \mathrm{C}_{6} .11$ \\
\hline 643 & & 638 & & $\begin{array}{l}\mathrm{C}_{12} \mathrm{O}_{13} \mathrm{H}_{43} .53, \mathrm{~N}_{21} \mathrm{C}_{3} \mathrm{C}_{4}-\mathrm{C}_{5} \mathrm{H}_{23} \mathrm{C}_{6} .19 \\
\mathrm{C}_{4} \mathrm{H}_{23} \mathrm{C}_{5}-\mathrm{C}_{6} \mathrm{C}_{7} \mathrm{~N}_{22} .11\end{array}$ \\
\hline 629 & & & 626 & $\mathrm{C}_{3} \mathrm{H}_{31} \mathrm{C}_{17}, \mathrm{C}_{18} \mathrm{H}_{32} \mathrm{H}_{33} .82, \mathrm{C}_{4} \mathrm{C}_{2} \mathrm{C}_{3}-\mathrm{C}_{17} \mathrm{H}_{31} \mathrm{C}_{18} .16$ \\
\hline 6611 & 610 & 610 & 601 & $\mathrm{~N}_{21}-\mathrm{C}_{4} .12, \mathrm{C}_{4} \mathrm{C}_{5} \mathrm{C}_{6} .11$ \\
\hline 576 & 585 & 580 & & $\mathrm{C}_{2} \mathrm{~N}_{21} \mathrm{C}_{1}-\mathrm{O}_{15} \mathrm{H}_{42} .85$ \\
\hline 548 & 555 & 560 & 555 & $\begin{array}{l}\mathrm{C}_{8} \mathrm{C}_{20} \mathrm{H}_{38} .24, \mathrm{C}_{11} \mathrm{C}_{20} \mathrm{H}_{38} .25, \mathrm{C}_{11} \mathrm{C}_{20} \mathrm{H}_{37} .18 \\
\mathrm{C}_{8} \mathrm{C}_{20} \mathrm{H}_{37} .14\end{array}$ \\
\hline 498 & 508 & 495 & 491 & $\mathrm{C}_{2} \mathrm{C}_{16} \mathrm{H}_{30} .16, \mathrm{C}_{3} \mathrm{C}_{17} \mathrm{C}_{18} .13, \mathrm{C}_{2} \mathrm{C}_{16} \mathrm{H}_{28} .11$ \\
\hline 473 & & 470 & 478 & $\mathrm{~N}_{22} \mathrm{C}_{8} \mathrm{C}_{9}-\mathrm{C}_{10} \mathrm{H}_{24} \mathrm{H}_{25} .69$ \\
\hline 458 & & & 457 & $\mathrm{C}_{1} \mathrm{~N}_{21} \mathrm{C}_{4} .13, \mathrm{~N}_{22} \mathrm{C}_{8} \mathrm{C}_{9}-\mathrm{C}_{10} \mathrm{H}_{24} \mathrm{H}_{25} .11$ \\
\hline 438 & & 430 & 432 & $\begin{array}{l}\mathrm{N}_{22} \mathrm{C}_{8} \mathrm{C}_{9}-\mathrm{C}_{10} \mathrm{H}_{24} \mathrm{H}_{25} .12, \mathrm{C}_{8} \mathrm{C}_{20} \mathrm{C}_{11} \cdot 16, \mathrm{C}_{20} \mathrm{C}_{11} \mathrm{C}_{12} \cdot 16 \\
\mathrm{C}_{11} \mathrm{O}_{14} \mathrm{C}_{12}-\mathrm{O}_{10} \mathrm{H}_{24} \mathrm{H}_{25} .13\end{array}$ \\
\hline 417 & & 415 & 416 & $\mathrm{C}_{1} \mathrm{C}_{3} \mathrm{C}_{2}-\mathrm{C}_{16} \mathrm{H}_{28} \mathrm{H}_{29} \mathrm{H}_{30} .37$ \\
\hline 394 & 392 & 390 & & $\mathrm{C}_{6} \mathrm{C}_{8} \mathrm{C}_{7} \mathrm{C}_{19} \mathrm{H}_{34} \mathrm{H}_{35} \mathrm{H}_{36} .23$ \\
\hline $\begin{array}{l}384 \\
344\end{array}$ & 378 & 385 & 383 & $\begin{array}{l}\mathrm{C}_{6} \mathrm{C}_{8} \mathrm{C}_{-}-\mathrm{C}_{19} \mathrm{H}_{34} \mathrm{H}_{35} \mathrm{H}_{36} \cdot 45, \mathrm{O}_{14} \mathrm{C}_{11} \mathrm{C}_{12}-\mathrm{O}_{13} \mathrm{H}_{43} \mathrm{H}_{44} \cdot 11 \\
\mathrm{O}_{15}-\mathrm{C}_{1} \mathrm{~N}_{21} \mathrm{C}_{2} .33, \mathrm{C}_{16}-\mathrm{C}_{2} \mathrm{C}_{1} \mathrm{C}_{3} .25 \\
\mathrm{C}_{1} \mathrm{C}_{3} \mathrm{C}_{2}-\mathrm{C}_{16} \mathrm{H}_{28} \mathrm{H}_{29} \mathrm{H}_{30} \cdot 11\end{array}$ \\
\hline 336 & & 330 & & $\mathrm{H}_{23} \mathrm{C}_{4} \mathrm{C}_{5}-\mathrm{C}_{6} \mathrm{C}_{7} \mathrm{~N}_{22} \cdot 14$ \\
\hline 303 & & 300 & 302 & $\mathrm{O}_{15}-\mathrm{H}_{42} .62$ \\
\hline
\end{tabular}


Table 3. continued

\begin{tabular}{|c|c|c|c|c|}
\hline \multirow[b]{2}{*}{ Calcd } & \multicolumn{2}{|c|}{ Raman } & \multirow[b]{2}{*}{ IR } & \multirow[b]{2}{*}{ Assignment and PED (\%) } \\
\hline & $\mathbf{R R}$ & FT-R & & \\
\hline 298 & 298 & & & $\mathrm{O}_{15}-\mathrm{H}_{44} .74$ \\
\hline 289 & 282 & 280 & 282 & $\mathrm{O}_{13}-\mathrm{H}_{43} .59, \mathrm{O}_{13}-\mathrm{H}_{44} .10$ \\
\hline 265 & 265 & 250 & 248 & $\begin{array}{l}\mathrm{C}_{3} \mathrm{C}_{4} \mathrm{C}_{2}-\mathrm{C}_{17} \mathrm{H}_{31} \mathrm{C}_{18} \cdot 16, \mathrm{O}_{15}-\mathrm{C}_{1} \mathrm{~N}_{21} \mathrm{C}_{2} .24 \\
\mathrm{C}_{1}-\mathrm{C}_{3} \mathrm{C}_{2} \mathrm{C}_{4} \cdot 14\end{array}$ \\
\hline 236 & 226 & & & $\mathrm{C}_{19}-\mathrm{C}_{7} \mathrm{C}_{6} \mathrm{C}_{8} .23, \mathrm{H}_{37} \mathrm{H}_{38} \mathrm{C}_{8} \mathrm{C}_{20}-\mathrm{C}_{11} \mathrm{C}_{12} \mathrm{H}_{39} \mathrm{H}_{40} .10$ \\
\hline 217 & & & 226 & $\mathrm{C}_{20}-\mathrm{C}_{8} \mathrm{C}_{9} \mathrm{C}_{7} .15, \mathrm{C}_{7} \mathrm{C}_{9} \mathrm{C}_{8}-\mathrm{C}_{20} \mathrm{C}_{11} \mathrm{H}_{37} \mathrm{H}_{38} .16$ \\
\hline 199 & 195 & 200 & 202 & $\mathrm{O}_{15} \mathrm{C}_{1} \mathrm{~N}_{21} .15, \mathrm{C}_{5}-\mathrm{C}_{6} .10$ \\
\hline 193 & 192 & & 187 & $\begin{array}{l}\mathrm{O}_{15} \mathrm{C}_{2} \mathrm{C}_{1}-\mathrm{N}_{21} \mathrm{C}_{4} \mathrm{H}_{26} .15, \mathrm{C}_{1} \mathrm{C}_{16} \mathrm{C}_{2}-\mathrm{C}_{3} \mathrm{C}_{17} \mathrm{C}_{4} .13 \\
\mathrm{C}_{17}-\mathrm{C}_{3} \mathrm{C}_{2} \mathrm{C}_{4} .10\end{array}$ \\
\hline 167 & 170 & & 171 & $\mathrm{C}_{14} \mathrm{C}_{7} \mathrm{C}_{\mathrm{x}} .23, \mathrm{C}_{10} \mathrm{C}_{9} \mathrm{~N}_{22} .29$ \\
\hline 156 & 160 & & 152 & $\mathrm{C}_{6} \mathrm{C}_{19} \mathrm{C}_{7}-\mathrm{C}_{8} \mathrm{C}_{20} \mathrm{C}_{9} .17, \mathrm{C}_{5} \mathrm{C}_{7} \mathrm{C}_{6}-\mathrm{N}_{22} \mathrm{C}_{9} \mathrm{H}_{27} .13$ \\
\hline 147 & & 145 & 145 & $\mathrm{C}_{10} \mathrm{C}_{9} \mathrm{C}_{8}-\mathrm{N}_{22} \mathrm{C}_{6} \mathrm{H}_{27} .11, \mathrm{C}_{16}-\mathrm{C}_{2} \mathrm{C}_{1} \mathrm{C}_{3} .10$ \\
\hline 140 & & & 136 & $\mathrm{C}_{16} \mathrm{C}_{2} \mathrm{C}_{1} .11, \mathrm{C}_{10} \mathrm{C}_{9} \mathrm{~N}_{22} .11$ \\
\hline 123 & & 122 & & $\mathrm{C}_{20} \mathrm{C}_{8} \mathrm{C}_{9} .12$ \\
\hline 115 & & 110 & 116 & $\mathrm{C}_{17} \mathrm{C}_{3} \mathrm{C}_{2} .14, \mathrm{H}_{27}-\mathrm{N}_{22} \mathrm{C}_{9} \mathrm{C}_{6} .10, \mathrm{C}_{5} \mathrm{C}_{7} .12$ \\
\hline 84 & & & 85 & $\mathrm{C}_{1} \mathrm{C}_{16} \mathrm{C}_{2}-\mathrm{C}_{3} \mathrm{C}_{17} \mathrm{C}_{4} .10, \mathrm{C}_{17} \mathrm{C}_{3} \mathrm{C}_{2} .15$ \\
\hline 79 & & & 85 & $\mathrm{C}_{12} \mathrm{O}_{13} \mathrm{H}_{43} .13, \mathrm{C}_{1} \mathrm{C}_{16} \mathrm{C}_{2}-\mathrm{C}_{3} \mathrm{C}_{17} \mathrm{C}_{4} .10$ \\
\hline 67 & & & 66 & $\mathrm{C}_{7} \mathrm{C}_{8} \mathrm{C}_{9}-\mathrm{C}_{20} \mathrm{C}_{11} \mathrm{H}_{37} \mathrm{H}_{38} .18, \mathrm{C}_{6} \mathrm{C}_{19} \mathrm{C}_{7}-\mathrm{C}_{8} \mathrm{C}_{20} \mathrm{C}_{9} .25$ \\
\hline 63 & & & 64 & $\mathrm{C}_{1} \mathrm{O}_{15} \mathrm{H}_{42} .92$ \\
\hline 49 & & & 47 & $\mathrm{C}_{12} \mathrm{O}_{13} \mathrm{H}_{43} .21, \mathrm{C}_{12} \mathrm{O}_{13} \mathrm{H}_{44} .35$ \\
\hline 31 & & & 31 & $\mathrm{H}_{31} \mathrm{C}_{17} \mathrm{C}_{3} .21, \mathrm{H}_{31} \mathrm{C}_{17} \mathrm{C}_{18} .21, \mathrm{C}_{3} \mathrm{C}_{17} \mathrm{C}_{18} .19$ \\
\hline
\end{tabular}

Raman). Eliminating hydrogen bonds changes the composition to vinyl, carbonyl bending and lactam bond stretching at $1161 \mathrm{~cm}^{-1}$ on the C-D ring. This band appears as a shoulder on the $1141 \mathrm{~cm}^{-1}$ band in vinylisoneoxanthobilirubinate spectra.

The A-B calculation of a pyrrole ring breathing mode at $1187 \mathrm{~cm}^{-1}$ is substantiated by the experimental evidence. Bilirubin IX $a$ and all of the model compounds with two pyrromethenone rings have a band at $1188-1192 \mathrm{~cm}^{-1}$ in solution Raman spectra. The band is observed at $1185-1186 \mathrm{~cm}^{-1}$ in spectra of solid bilirubin IX $\alpha$ and at $1196-1198 \mathrm{~cm}^{-1}$ in the half-molecules. The C-D ring calculation predicts this band at $1181 \mathrm{~cm}^{-1}$, a mixture of pyrrole and lactam ring stretches. That there is almost no hydrogen bond sensitivity observed makes this assignment less likely.

The $1200-1300 \mathrm{~cm}^{-1}$ region contains several closely spaced and environment-sensitive bands, mostly localized on the lactam moieties. The relative intensities are quite different in resonance Raman and IR spectra. In bilirubin IX $a$ itself the bands are incompletely resolved, and may appear as shoulders or as single broad bands.

The hydrogen-bonded model predicts a lactam-A mode at $1225 \mathrm{~cm}^{-1}$ and assigns it to coupled $\mathrm{C}-\mathrm{C}$ stretches on the lactam ring and vinyl side chain bending. This band can be equated to the $1220 \mathrm{~cm}^{-1}$ band in IR spectra. It is very weak in the Raman spectra of hydrogen-bonded molecules. The simplified model predicts no corresponding mode near this region; however, we do observe a shoulder at $c a 1221 \mathrm{~cm}^{-1}$ in Raman spectra of the bilirubin monomer.

The resonance Raman spectra of bilirubin IX $\alpha$, III $\alpha$ and XIII $\alpha$ all contain at least three bands at $c a 1250,1270$ and $1285 \mathrm{~cm}^{-1}$. The relative intensities are strongly environment-sensitive and the bands are not always clearly resolved. A propionic acid carbon hydrogen bending mode is calculated (hydrogen-bonded structure) at $1243 \mathrm{~cm}^{-1}$, and may be a component of the strong IR band at $1250 \mathrm{~cm}^{-1}$. However, this vibration cannot contribute to the strong resonance Raman intensity observed near $1250 \mathrm{~cm}^{-1}$ in BR IX $\alpha$ and model compounds.

The non-hydrogen-bonded model finds a lactam ring, carbonyl and vinyl group deformation at $1266 \mathrm{~cm}^{-1}$ on lactam $\mathrm{D}$ and a lactam $\mathrm{D}$ stretching mode at $1278 \mathrm{~cm}^{-1}$. These bands are detected in the bilirubin half molecule and bilirubin-lipid complexes as strong and broad bands at $c a 1268-1276 \mathrm{~cm}^{-1}$. Raman bands at this region are strongly 
Table 4. Frequencies and normal coordinate analysis of bilirubin in the range $900-1700 \mathrm{~cm}^{-1}$ (CD pyrromethenone)

\begin{tabular}{|c|c|c|c|}
\hline Calculated & Raman & Coordinates & Assignment \\
\hline 1647 & & $\begin{array}{l}18.3 \% \\
\mathrm{C}_{5}-\mathrm{C}_{9}, \mathrm{C}_{15}-\mathrm{C}_{19}, \mathrm{C}_{14-} \\
\mathrm{C}_{21}, \mathrm{C}_{4}=\mathrm{C}_{11}, \mathrm{C}_{15}- \\
\mathrm{N}_{16}, \mathrm{C}_{13}-\mathrm{C}_{14}, \mathrm{C}_{2}-\mathrm{C}_{3}\end{array}$ & $\begin{array}{l}\text { Vinyl coupled lactam and } \\
\text { pyrrole ring stretching }\end{array}$ \\
\hline 1603 & 1598 & $\begin{array}{l}34.3 \% \\
\mathrm{C}_{14} \mathrm{C}_{13} \mathrm{C}_{14}, \mathrm{C}_{18} \mathrm{C}_{13} \mathrm{C}_{12} \\
\mathrm{C}_{13} \mathrm{C}_{12} \mathrm{~N}_{16}, \mathrm{C}_{14} \mathrm{C}_{14} \mathrm{C}_{13} \\
\mathrm{C}_{14}=\mathrm{C}_{15}, \mathrm{C}_{12}-\mathrm{N}_{16}\end{array}$ & Pyrrole ring deformation \\
\hline 1568 & 1567 & $\begin{array}{l}24.4 \% \\
C_{4}=C_{11}, C_{21}-C_{22}, C_{1}- \\
C_{2}, C_{19}-C_{21}, C_{4}-C_{4}, C_{15}- \\
C_{19}, C_{15}-N_{16}, C_{5}-N_{6}, C_{1}- \\
C_{12}, C_{3}=C_{4}\end{array}$ & $\begin{array}{l}\text { Vinyl, lactam } C=C \text {, } \\
\text { bridge } C=C \text { stretching }\end{array}$ \\
\hline 1552 & 1512 & $\begin{array}{l}10.5 \% \\
\mathrm{~N}_{16}-\mathrm{H}_{17}, \mathrm{C}_{14}=\mathrm{C}_{15}, \mathrm{C}_{21}- \\
\mathrm{C}_{22}, \mathrm{C}_{12}=\mathrm{C}_{13}, \mathrm{C}_{5}=\mathrm{O}_{111}, \\
\mathrm{C}_{-}-\mathrm{N}_{6} \\
51.8 \%\end{array}$ & $\begin{array}{l}\text { Pyrrole } \mathrm{C}=\mathrm{C}, \mathrm{N}-\mathrm{H} \text { and } \\
\text { lactam } \mathrm{C}=\mathrm{O}, \mathrm{C}-\mathrm{N} \\
\text { stretching }\end{array}$ \\
\hline & & $\begin{array}{l}\mathrm{C}_{2} \mathrm{C}_{3}=\mathrm{C}_{4} \mathrm{C}_{5}, \mathrm{~N}_{6} \mathrm{C}_{2}- \\
\mathrm{C}_{3} \mathrm{C}_{4}, \mathrm{C}_{6}-\mathrm{C}_{3} \mathrm{C}_{2} \mathrm{C}_{4}, \mathrm{C}_{4}- \\
\mathrm{C}_{4} \mathrm{C}_{3} \mathrm{C}_{5}, \mathrm{C}_{1}-\mathrm{C}_{2} \mathrm{C}_{3} \mathrm{~N}_{6}\end{array}$ & Lactam ring torsion \\
\hline 1461 & 1467 & $\begin{array}{l}26.7 \% \\
C_{2} C_{1} C_{12}, C_{1} C_{12} C_{13}, C_{1} \\
C_{2} C_{3}, C_{1} C_{2} N_{6}, C_{12} C_{13} \\
C_{14}, C_{1} C_{12} N_{16}\end{array}$ & $\begin{array}{l}\text { Bridge } \mathrm{C}-\mathrm{C}, \mathrm{C}=\mathrm{C} \\
\text { deformation }\end{array}$ \\
\hline 1440 & 1443 & $\begin{array}{l}74.5 \% \\
\mathrm{C}_{12} \mathrm{C}_{15} \\
\mathrm{C}_{14} \mathrm{C}_{15}, \mathrm{C}_{13} \mathrm{C}_{14}=\mathrm{C}_{15} \mathrm{~N}_{16}, \\
\mathrm{C}_{15}-\mathrm{C}_{13} \mathrm{C}_{12} \mathrm{C}_{14}\end{array}$ & Pyrrole ring deformation \\
\hline 1427 & & $\begin{array}{l}26.3 \% \\
\mathrm{C}_{13} \mathrm{C}_{14} \mathrm{C}_{15}, \mathrm{O}_{10} \mathrm{C}_{5} \mathrm{C}_{4}, \mathrm{C}_{21} \\
\mathrm{C}_{15} \mathrm{C}_{14}, \mathrm{C}_{4} \mathrm{C}_{4} \mathrm{C}_{5}, \mathrm{C}_{19} \\
\mathrm{C}_{14} \mathrm{C}_{15}, \mathrm{C}_{19} \mathrm{C}_{14} \mathrm{~N}_{16}, \mathrm{C}_{3} \\
\mathrm{C}_{4} \mathrm{C}_{5}, \mathrm{C}_{2} \mathrm{C}_{1} \mathrm{C}_{12}\end{array}$ & $\begin{array}{l}\text { Deformation of } \mathrm{C}=\mathrm{O} \text { and } \\
\text { methyl groups on lactam } \\
\text { and pyrrole }\end{array}$ \\
\hline 1329 & 1337 & $\begin{array}{l}48.7 \% \\
\mathrm{C}_{3} \mathrm{C}_{4}-\mathrm{C}_{5} \mathrm{~N}_{6}, \mathrm{O}_{10}- \\
\mathrm{C}_{5} \mathrm{C}_{4} \mathrm{~N}_{6}, \mathrm{C}_{4} \mathrm{C}_{5}- \\
\mathrm{N}_{6} \mathrm{C}_{2}, \mathrm{C}_{4}-\mathrm{C}_{4} \mathrm{C}_{3} \mathrm{C}_{5}\end{array}$ & Lactam torsion \\
\hline 1322 & 1319 & $\begin{array}{l}26.3 \% \\
\mathrm{C}_{1,5}-\mathrm{N}_{16}, \mathrm{C}_{1}-\mathrm{C}_{12}, \mathrm{C}_{1}- \\
\mathrm{C}_{2}, \mathrm{C}_{4}-\mathrm{C}_{4}, \mathrm{C}_{14}-\mathrm{C}_{19}, \mathrm{C}_{5}- \\
\mathrm{N}_{6}, \mathrm{C}_{2}-\mathrm{C}_{3}\end{array}$ & $\begin{array}{l}\text { Pyrrole, lactam ring and } \\
\text { bridge stretching }\end{array}$ \\
\hline 1309 & & $\begin{array}{l}13.7 \% \\
\mathrm{C}_{5}-\mathrm{C}_{9}, \mathrm{C}_{15} \\
\mathrm{C}_{19}, \mathrm{C}_{9}=\mathrm{C}_{11}, \mathrm{C}_{15} \\
\mathrm{~N}_{16}, \mathrm{C}_{13}-\mathrm{C}_{14}, \mathrm{C}_{21^{-}} \\
\mathrm{C}_{22}, \mathrm{C}_{2}-\mathrm{C}_{3}, \mathrm{C}_{5}-\mathrm{N}_{6}\end{array}$ & $\begin{array}{l}\text { Vinyl coupled lactam and } \\
\text { pyrrole stretching }\end{array}$ \\
\hline 1278 & 1274 & $\begin{array}{l}18.3 \% \\
\mathrm{C}_{15}-\mathrm{C}_{21}, \mathrm{C}_{1}-\mathrm{C}_{9}, \mathrm{C}_{21}- \\
\mathrm{C}_{22}, \mathrm{C}_{1}-\mathrm{C}_{12}, \mathrm{C}_{19}=\mathrm{C}_{11}, \mathrm{~N}_{6-} \\
\mathrm{H}_{7}, \mathrm{C}_{1}-\mathrm{C}_{2}\end{array}$ & $\begin{array}{l}\text { Vinyl coupled, bridge } \\
\mathrm{C}=\mathrm{C} \text {, and lactam } \mathrm{N}-\mathrm{H} \\
\text { stretching }\end{array}$ \\
\hline 1266 & 1244 & $\begin{array}{l}24.0 \% \\
\mathrm{C}_{3} \mathrm{~N}_{6} \mathrm{C}_{5}, \mathrm{C}_{2} \mathrm{C}_{3} \mathrm{C}_{4}, \mathrm{C}_{9} \mathrm{C}_{4} \\
\mathrm{C}_{3}, \mathrm{C}_{3} \mathrm{C}_{2} \mathrm{~N}_{6}, \mathrm{O}_{10} \mathrm{C}_{5} \mathrm{~N}_{6}\end{array}$ & $\begin{array}{l}\text { Lactam ring and carbonyl } \\
\text { deformation }\end{array}$ \\
\hline
\end{tabular}


Table 4. continued

\begin{tabular}{|c|c|c|c|}
\hline Calculated & Raman & Coordinates & Assignment \\
\hline 1181 & 1198 & $\begin{array}{l}15.9 \% \\
C_{9}=C_{11}, C_{21}-C_{22}, C_{1}- \\
C_{2}, C_{19}-C_{21}\end{array}$ & $\begin{array}{l}\text { Vinyl and bridge } \mathrm{C}=\mathrm{C} \\
\text { stretching }\end{array}$ \\
\hline 1144 & 1141 & $\begin{array}{l}42.4 \% \\
C_{22} C_{21} C_{14}, C_{21} C_{14} C_{14}, \\
C_{14} C_{13} C_{14}, C_{24} C_{15} C_{14}, \\
C_{21}-C_{22}, C_{13}-C_{14}\end{array}$ & $\begin{array}{l}\text { Deformation of methyl } \\
\text { group on pyrrole ring }\end{array}$ \\
\hline 1161 & & $\begin{array}{l}24.1 \% \\
\mathrm{C}_{11} \mathrm{C}_{9} \mathrm{C}_{4}, \mathrm{O}_{10} \mathrm{C}_{5} \mathrm{C}_{4}, \mathrm{C}_{8} \\
\mathrm{C}_{3} \mathrm{C}_{4}, \mathrm{C}_{5}-\mathrm{N}_{6}, \mathrm{C}_{2}-\mathrm{C}_{3}\end{array}$ & $\begin{array}{l}\text { Deformation and bond } \\
\text { stretching of lactam ring }\end{array}$ \\
\hline 1107 & & $\begin{array}{l}18.2 \% \\
C_{4}=C_{11}, C_{21}-C_{22}, C_{1}- \\
C_{2}, C_{15}-C_{21}, C_{5}-N_{6}, C_{15} \\
N_{16}\end{array}$ & $\begin{array}{l}\text { Vinyl and bridge } \mathrm{C}=\mathrm{C} \\
\text { coupled } \mathrm{C}-\mathrm{N} \text { stretching }\end{array}$ \\
\hline 1072 & 1055 & $\begin{array}{l}10.8 \% \\
\mathrm{~N}_{16}-\mathrm{H}_{17}, \mathrm{C}_{14}=\mathrm{C}_{15}, \mathrm{C}_{21}- \\
\mathrm{C}_{22}, \mathrm{C}_{12}=\mathrm{C}_{13}, \mathrm{C}_{5}=\mathrm{O}_{11}, \\
\mathrm{C}_{2}-\mathrm{N}_{6}\end{array}$ & $\begin{array}{l}\text { Pyrrole } \mathrm{C}=\mathrm{C}, \mathrm{N}-\mathrm{H} \text {, lactam } \\
\mathrm{C}-\mathrm{N} \text { and } \mathrm{C}=\mathrm{O} \text { stretching }\end{array}$ \\
\hline 1032 & 993 & $\begin{array}{l}92.15 \% \\
N_{16}-C_{12} \\
C_{1} C_{2}, N_{6} C_{2}=C_{1} C_{12}, C_{6}- \\
C_{3} C_{2} C_{4}, C_{5} N_{6}-C_{2} C_{3}\end{array}$ & $\begin{array}{l}\text { Bridge } \mathrm{C}=\mathrm{C} \text { and } \mathrm{C}-\mathrm{C} \\
\text { torsion, lactam } \mathrm{C}-\mathrm{N} \\
\text { torsion }\end{array}$ \\
\hline 945 & & $\begin{array}{l}46.4 \% \\
C_{15}-C_{211}, C_{13}-C_{18}, C_{1}- \\
C_{12}, C_{2}-C_{3}, C_{9}=C_{11}, \\
N_{6}-H_{7}\end{array}$ & $\begin{array}{l}\text { Bridge } \mathrm{C}-\mathrm{C} \text {, vinyl, lactam } \\
\mathrm{N}-\mathrm{H} \text { stretch }\end{array}$ \\
\hline
\end{tabular}

environmental sensitive. Therefore the assignment to lactum ring modes is realistic; however, neither set of calculations predicts the presence of three bands in this region. While this may be a defect of the models, it is also possible that there is Fermi resonance with the overtone of one of the bands in the $610-640 \mathrm{~cm}^{-1}$ region.

The A-B model assigns a bridge $\mathrm{C}-\mathrm{H}$ bending, bridge $\mathrm{C}=\mathrm{C}$ stretch, and lactam- $\mathrm{A}$ $\mathrm{C}-\mathrm{N}$ stretch to $1310 \mathrm{~cm}^{-1}$. There are weak bands in the IR $\left(1301 \mathrm{~cm}^{-1}\right)$ and Raman which

Table 5. Comparison of selected bands $\left(\mathrm{cm}^{-1}\right)$ from the spectra of $\mathrm{D}_{2} \mathrm{BR}-d^{6}$ and $\mathrm{H}_{2} \mathrm{BR}$ in fluorolube

\begin{tabular}{lccccc}
\hline Group & $\begin{array}{c}\mathrm{D}_{2} \mathrm{BR}-d^{6} \\
\text { Calculated }\end{array}$ & Observed & $\begin{array}{c}\mathrm{H}_{2} \mathrm{BR}^{*} \\
\text { (in D }\end{array}$ & $\begin{array}{c}\left.\mathrm{H}_{2} \mathrm{BR}\right) \\
\text { Calculated }\end{array}$ & Observed \\
\hline NH pyrrole & 2499 & 2541 & 3407 & 3409 & 3410 \\
& & 2444 & & & \\
NH lactam & 2395 & 2417 & 3261 & 3060 & 3262 \\
CH stretch & 3100 & 3081 & & 3100 & 3101 \\
CH stretch & 3070 & 3061 & & 3070 & 3070 \\
CH stretch & 3002 & 3011 & & 3002 & 3011 \\
C=O & 1693 & 1700 & & 1693 & 1692 \\
C=C & 1655 & 1640 & & 1655 & 1649 \\
C=O & & & & & \\
N-C & 1599 & 1610 & & 1606 & 1612 \\
C=O & & & & & \\
C=C & & & & & \\
N-C & & & & & \\
\hline
\end{tabular}

\footnotetext{
* Some $\mathrm{H}_{2} \mathrm{BR}$ remains in the spectrum despite the careful sample preparations.
} 
may be this mode. The C-D model predicts a similar vibration at 1309 and $1322 \mathrm{~cm}^{-1}$ for pyrrole-lactam ring stretches. These bands are detected at $1319 \mathrm{~cm}^{-1}$ as medium-tostrong bands in vinylisoneoxanthobilirubinate aqueous solution.

The C-D model also predicts a lactam ring torsion mode at $1329 \mathrm{~cm}^{-1}$. This may be the weak band at $1337 \mathrm{~cm}^{-1}$ in the vinylisoneoxanthobilirubinate spectrum. We have previously observed that in the spectra of bilirubin and its model compounds (BR III $a$, BR XIII $\alpha$ ) this band (at $c a 1344 \mathrm{~cm}^{-1}$ ) is sensitive to the vinyl group but insensitive to hydrogen bonding on lactam carbonyl groups. The A-B calculation of methyl $\mathrm{C}-\mathrm{H}$ bending modes at 1353 and $1356 \mathrm{~cm}^{-1}$ may describe the IR spectra adequately, but cannot account for the strong resonance Raman band arising from the $\mathrm{A}-\mathrm{B}$ ring at $\mathrm{ca}$ $1344 \mathrm{~cm}^{-1}$. We find it hard to evaluate this assignment conclusively.

The A-B model calculation of a coupled lactam C-N stretch, methyl $\mathrm{C}-\mathrm{H}$ bending mode at $1395 \mathrm{~cm}^{-1}$ fits the observed Raman $\left(1395 \mathrm{~cm}^{-1}\right)$ and IR $\left(1405 \mathrm{~cm}^{-1}\right)$ data well. The C-D model calculation does not contain a similar mode because it does not include the methyl hydrogens.

The non-hydrogen-bonded model calculation shows three bands in the $1427-1460 \mathrm{~cm}^{-1}$ region. The pyrrole-C torsion is predicted to be at $1440 \mathrm{~cm}^{-1}$. A C-D pyrrole-lactam bridging carbon deformation is predicted at $1461 \mathrm{~cm}^{-1}$. These two bands correspond to the two hydrogen-bond-sensitive bands at 1443 and $1467 \mathrm{~cm}^{-1}$, respectively, in the Raman spectrum of vinylisoneoxanthobilirubinate.

A vinyl $\mathrm{C}-\mathrm{H}$ bending mode is calculated (A-B) at $1487 \mathrm{~cm}^{-1}$, and is identified with the $1500 \mathrm{~cm}^{-1}$ IR band. The simplified C-D model predicts a lactam $D$ torsional motion at $1495 \mathrm{~cm}^{-1}$. This mode can be equated to the $1512 \mathrm{~cm}^{-1}$ band in the vinylisoneoxanthobilirubinate solution spectrum.

The A-B model calculates two bands at 1567 and $1606 \mathrm{~cm}^{-1}$. The IR spectra fit this prediction well. The assignment of a lactam $\mathrm{C}=\mathrm{C}, \mathrm{C}-\mathrm{N}, \mathrm{C}=\mathrm{O}$, pyrrole $\mathrm{C}=\mathrm{C}$ stretch is reasonable for the band at $c a 1605-1616 \mathrm{~cm}^{-1}$ observed in bilirubin IX $a$ and other model compounds which have hydrogen bonds. Similarly, the predicted $1567 \mathrm{~cm}^{-1}$ backbone stretching mode agrees well with the IR spectra and the solution Raman spectra of acidic forms of bilirubin derivatives. The hydrogen bond sensitivity of this band is consistent with the mode composition.

The C-D model predicts three bands in the $1545-1610 \mathrm{~cm}^{-1}$ region. The $1552 \mathrm{~cm}^{-1}$ band is weak and not well resolved in the Raman spectrum of vinylisoneoxanthobilirubinate. Bands at 1568 and $1603 \mathrm{~cm}^{-1}$ are equated to the weak band at 1567 and a strong band at $1598 \mathrm{~cm}^{-1}$, respectively. The band at $1567 \mathrm{~cm}^{-1}$ is calculated to have a similar assignment as in the A-B H-bonded model; however, the $1603 \mathrm{~cm}^{-1}$ band is calculated to be a pyrrole-localized vibration. This is characteristic for non-hydrogen-bonded bilirubins. For example, we observe this band at 1597,1601 and $1598 \mathrm{~cm}^{-1}$ in bilirubin $\mathrm{IXa} /$ sphingomyelin, bilirubin IIIa/sphingomyelin complex and exovinylneoxatho bilirubinate spectra. This band is obscured by the strong and characteristic $1615 \mathrm{~cm}^{-1}$ band of the intramolecularly hydrogen-bonded molecules (BR IIIa, BR IXa, etc.)

Both sets of calculations agree well on the carbonyl stretches at $c a 1650 \mathrm{~cm}^{-1}$. We have examined the Raman spectra of the $\alpha, \alpha^{\prime}$-dimethyl MBR (meso) (Fig. 3) and $\alpha, \alpha^{\prime}$ dimethyl MBR (racemic) compounds in chloroform (Fig. 4). The only differences between these two compounds are the orientation of the methyl groups which are in the $\alpha$ position with respect to the carboxylate group. By structural predictions [12] there should be no appreciable differences between the racemic compound and nonsubstituted MBR XIIIa, while the meso molecule should show steric hindrance from the methyl group, especially in the lactam moiety. In chloroform solvent, where six hydrogen bonds are formed, we observe the band shifting from 1644 to $1648 \mathrm{~cm}^{-1}$. This confirms that the $1644 \mathrm{~cm}^{-1}$ band involves largely the lactam ring vibration.

Bands from 2000 to $4000 \mathrm{~cm}^{-1}$ have been largely assigned to $\mathrm{N}-\mathrm{H}$ and $\mathrm{C}-\mathrm{H}$ stretching. The normal coordinate analysis provides clear answers to some previously posed questions and offers reasonable explanations for the experimental results. For example, in the IR spectrum for the acid salt of calcium bilirubinate $(\% \mathrm{Ca}=3.2)$, the relative intensity of the $3410 \mathrm{~cm}^{-1}$ bands does change when compared with that of protonated 


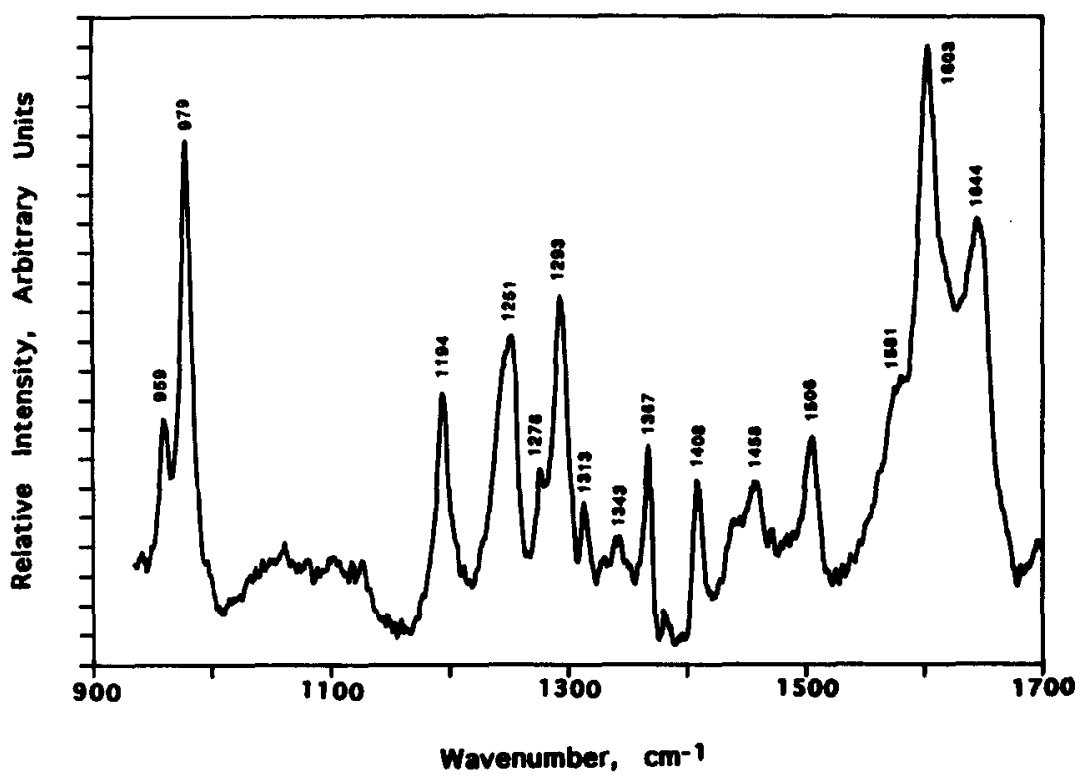

Fig. 3. Resonance Raman spectrum of $5 \times 10^{-4} \mathrm{M} \alpha, \alpha^{\prime}$-dimethyl MBR XIII $\alpha$ (meso) in chloroform; $532 \mathrm{~nm}$ excitation.

bilirubin. Thus the $3410 \mathrm{~cm}^{-1}$ band cannot be assigned to the $\mathrm{OH}$ group. Our calculations indicate that this band is due to the stretching mode of the $\mathrm{N}-\mathrm{H}$ vibration. The observed result provides evidence that the $\mathbf{N}-\mathbf{H}$ group coordinates with calcium.

Table 5 shows that in perdeuterated bilirubin, the $3410 \mathrm{~cm}^{-1}$ peak of protonated bilirubin, representing the pyrrole $\mathrm{N}-\mathrm{H}$, shifts to $2541 \mathrm{~cm}^{-1}$, and the $3260 \mathrm{~cm}^{-1}$ peak, assigned as the lactam $\mathrm{N}-\mathrm{H}$, shifts to $2444 \mathrm{~cm}^{-1}$. That the peak intensities of the 2541 and $2444 \mathrm{~cm}^{-1}$ are more than four times the intensities of the original peaks indicates that most of the N-hydrogens have been replaced by deuterium. Broderson et al. [23] obtained similar peak shifts for the pyrrole but not for the lactam bands and found that the peak heights for the shifted bands were about equivalent to the original peaks. He

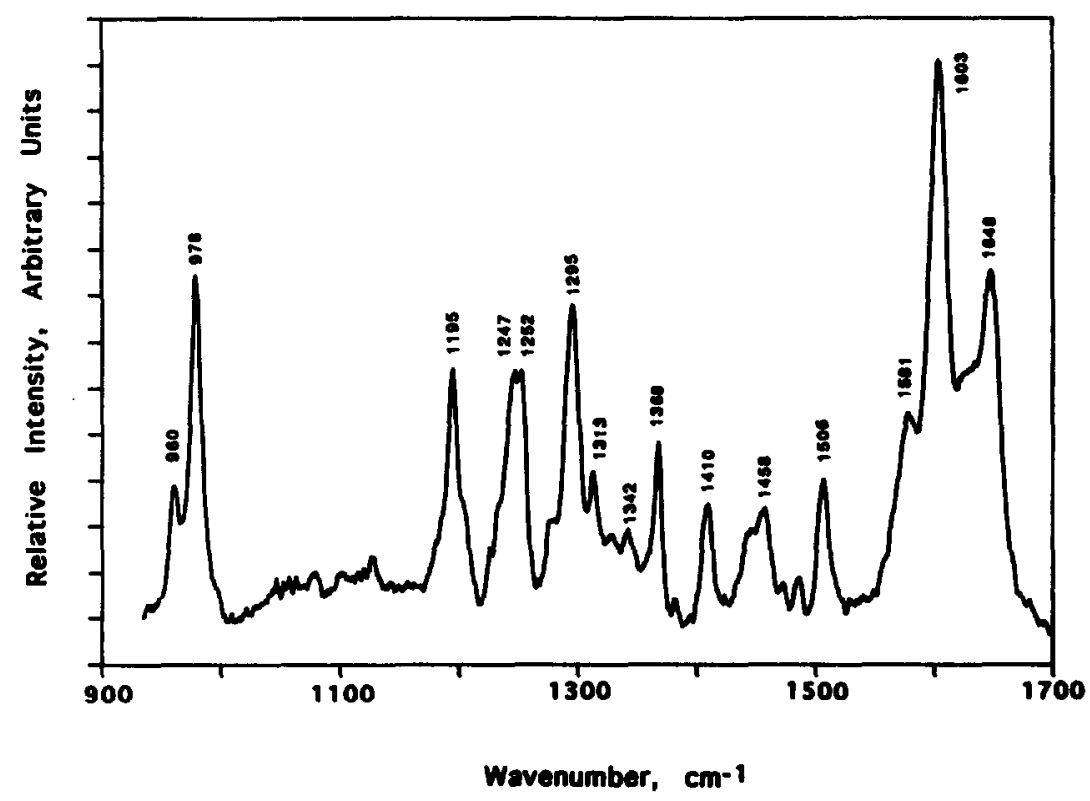

Fig. 4. Resonance Raman spectrum of $5 \times 10^{-4} \mathrm{~m} \alpha, \alpha^{\prime}$-dimethyl MBR XIlla (racemic) in chloroform; $532 \mathrm{~nm}$ excitation. 
concluded that deuterated bilirubin contained only four active hydrogens, two from the carboxyl groups and two from the four nitrogen groups.

Our results, however, indicate that both the pyrrole and lactam groups shift independently as indicated by the relative intensities of the shifted bands. There are several factors which make those band shifts detectable in our experiments. Firstly, our experiments were performed under improved experimental conditions, which permit incorporation of more deuterium. Secondly, we were able to examine the deuterated bilirubin without the need for desiccation. For that we used a fluorolube mull instead of $\mathrm{KBr}$ pellets. This process eliminates the trace water in desiccated $\mathrm{KBr}$ which undergoes re-exchange with deuterated bilirubin, reducing the proportion of deuteration. In fact, when we made a $\mathrm{KBr}$ pellet of $\mathrm{D}_{2} \mathrm{BR}$, we obtained results similar to those of BRODERSON et al. [23], in that the $2444 \mathrm{~cm}^{-1}$ peak appeared only as a shoulder. Therefore we conclude, and find independent support in the NMR studies of KAPLAN and NAVON [24], that the lactam deuterium may be more active than the pyrrole deuterium, preferentially eliminating this band during pellet formation and explaining why Broderson did not detect the $2444 \mathrm{~cm}^{-1}$ band. It is clear that both the previous [23] and present studies support the band assignments proposed in our model.

\section{CONCLUSION}

The evaluation of the force field of a molecule as complicated as a bilirubin pyrromethenone is by no means perfect. However, with reasonable molecular models and a realistic potential field, we can make accurate predictions about bilirubins containing or lacking intramolecular hydrogen bonds. Our normal coordinate analysis provides reasonable band assignments for IR Raman bands in the most interesting spectral regions. In addition, the results have clarified a number of obscure or mistaken concepts concerning the spectral and chemical properties of bilirubin.

Acknowledgements-We wish to thank David A. Lightner for providing samples of $\alpha, a^{\prime}$ dimethylmesobilirubin (MBR) in the meso and racemic forms. This work was supported in part by NIH grants NS-26160 (to M.D.M.) and AM-16549 (to R.D.S.) and by the State Commission of Education of China (to J.-G.W.).

\section{REFERENCES}

[1] J.-G. Wu, R. D. Soloway, D.-F. Xu, X.-Z. Wang, D. K. Martini and A. J. Wagner, Proc. SPIE 1145, 264 (1989).

[2] H. Guo, R. D. Soloway, Z. Yang, X.-C. Ding, D.-F. Xu and J.-G. Wu, Proc. SPIE 1145, 413 (1989).

[3] R. D. Soloway, J.-G. Wu, D.-F. Xu, Y.-F. Zhang, D. K. Martini, N.-K. Hong and R. S. Crowther, Proc. SPIE 1145, 262 (1989).

[4] H. Ishida, R. Kamoto, S. Uchida, A. Ishitani, Y. Ozaki, K. Iriyama, E. Tsukie, K. Shibata, F. Ishihara and H. Kameda, Appl. Spectrosc. 41, 407 (1987).

[5] S. Zheng and A. T. Tu, Appl. Spectrosc. 41, 696 (1987).

[6] Y.-Z. Hsieh and M. D. Morris, J. Am. Chem. Soc. 110, 62 (1988).

[7] B. Yang, M. D. Morris, M. Xie and D. A. Lightner, Biochemistry 30, 688 (1991).

[8] Y.-Z. Hsieh, N.-S. Lee, R.-S. Sheng and M. D. Morris, Langmuir 3, 1441 (1991).

[9] A. F. McDonagh, in The Porphyrins (Edited by D. Dolphin), Vol. VI, pp. 293-491. Academic Press, London (1973).

[10] L. Margulies and M. Toporowicz, J. Molec. Struct. 174, 153 (1988).

[11] X.-Z. Wang, R. D. Soloway, J.-G. Wu, B.-Z. Yu and G.-X. Xu, Proc. SPIE 1145, 132 (1989).

[12] G. Puzicha, Y.-M. Pu and D. A. Lightner, J. Am. Chem. Soc. 113, 3583 (1991).

[13] R. Bonnett, J. E. Davies, M. B. Hursthouse and G. M. Sheldrick, Proc. R. Soc. Lond. (Biol.) 202,249 (1978).

[14] D. P. McDermott, J. Phys. Chem. 90, 2569 (1986).

[15] D. W. Scott, J. Molec. Spectrosc. 31, 451 (1969).

[16] D. W. Scott, J. Molec. Spectrosc. 37, 77 (1971).

[17] P. Császár and A. Császár, J. Molec. Struct. 136, 323 (1986).

[18] G. C. Pimentel and A. L. McClellan, The Hydrogen Bond. W. H. Freeman, San Francisco (1960).

[19] E. B. Wilson, J. C. Decius and P. C. Cross, Molecular Vibrations. Dover, New York (1955). 
[20] C. Califano, Vibrational States. John Wiley, London (1976).

[21] J. H. Schatschneider, Reports 231/64 and 57/65, Shell Development Co., West Hollow Research Center, P.O. Box 1380, Houston, Texas (1965).

[22] D. F. McIntosh and M. R. Peterson, Quantum Chemistry Program Exchange, Program 342 (1977).

[23] R. Broderson, H. Flodgaard and J. K. Hansen, Acta. Chem. Scand. 21, 2284 (1967).

[24] D. Kaplan and G. Navon, J. Chem. Soc. Perkin Trans. 2, 1374 (1981). 